\title{
BACK PROPAGATION NEURAL NETWORKS FOR PREDICTING ULTIMATE STRENGTHS OF UNIDIRECTIONAL GRAPHITE/EPOXY TENSILE SPECIMENS
}

\author{
James L. Walker \\ University of Alabama in Huntsville \\ Deparment of Mechanical Engineering \\ Huntsville, AL 35899 \\ 205-828-3128
}

\author{
Eric v. K. Hill \\ Embry-Riddle Aeronautical University \\ Aerospace Engineering Deparment \\ Daytona Beach, FL 32114 \\ 904-226-6760
}

\section{ABSTRACT}

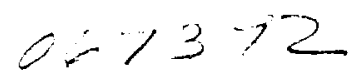

The research presented herein demonstrates the feasibility of predicting ultimate strengths in simple composite structures through a neural network analysis of their acoustic emission (AE) amplitude distribution data. A series of eleven ASTM D-3039 unidirectional graphite/epoxy tensile samples were loaded to failure to generate the amplitude distributions for this analysis. A back propagation neural network was trained to correlate the AE amplitude distribution signatures generated during the first $25 \%$ of loading with the ultimate strengths of the samples. The network was trained using two sets of inputs: (1) the statistical parameters obtained from a Weibull distribution fit of the amplinde distribution data, and (2) the event frequency (amplitude) distribution itself. The neural networks were able to predict ultimate strengths with a worst case error of $-8.99 \%$ for the Weibull modeled amplitude distribution data and $3.74 \%$ when the amplitude aistribution itself was used to tain the network. The principal reason for the improved prediction capability of the latter technique lies in the ability of the neural network to extract subtle features from within the ampiitude distribution.

\subsection{INTRODUCTION}

Previous research [1] has indicated that ultimate stengths could be predicted by mathematically modeling the amplitude aistribution of composite tensile specimens with a Weibull distribution. The analysis demonstrated that an equation of the form $\sigma_{u}=C_{0}+C_{1} b+C_{2} \theta+C_{3} b^{*} \theta$ could be used to predict ultimate strengths, where " $\mathrm{b}$ " and " $\theta$ " are the Weibull distribution shape parameters and the density function is given by $f(x)=(b / \theta) *(A / \theta)^{b-1} * \exp (A / \theta)^{b}$. The Weibull parameters were therefore proposed as inputs to a back propagation neural network. Research has also demonstrated [2] that a back propagation neural network model of the $\mathrm{AE}$ amplitude data collected during the initial stages of loading of 2195 aluminum-lithium alloy weldments could be used to predict their ultimate strength. There the number of $A E$ hits recorded at $1 \mathrm{~dB}$ intervals were used as the input vectors to the neural network. It was thought that a similar approach might work with these composite tensile coupons.

\subsection{NEURAL NETWORK ANALYSIS}

NeuralWorks Professional II/PLUS software, by NeuralWare, Inc., was used to develop the back propagation neural networks for this paper. Input data was fed into the network through an array of input neurons. Each input neuron was then fully connected by a series of weighting functions to a layer of hidden neurons and these in turn were fully connected to the output neuron. A bias neuron was weight connected to the hidden and output layer neurons to serve as a constant reference or offset value in the network. 
The weighting functions serve as the memory of a trained network by providing a multiplier between a preceding neuron's output value and an ensuing neuron's input value. A back propagation neural network works by minimizing the error between the generated (neural) output and the desired (actual) output using a gradient descent approach. Randomized weights are initially given to the interconnections of the network and an ourput value is calculated in response to an input data set. An error is then calculated and normalized so that a transfer function can determine the change, or delta, to back propagate to each of the connections in the network. This process is then repeated until the error reaches a desired
threshold.

Six AE data sets were generated by loading samples at a rate of $500 \mathrm{lbs} /$ minute to failure, while the $A E$ activity was monitored with a single Physical Acoustics Corporation (PAC) R15 tansducer and PAC LOCAN-AT. Only the portion of the AE amplitude data collected up to $25 \%(1500 \mathrm{lbs})$ of the expected failure load were supplied as inputs to the statistical analysis and neural network models. Five additional samples were loaded to failure and analyzed separately to provide a test base for the ultimate strength

The first neural network was trained using the Weibull parameters of the modeled amplitude distribution as inputs to demonstrate any similarities or differences with the results of the previous multivariate statistical analysis. A two layer network consisting of only an input and output layer was used, since it was known from the previous experimental work [1] that the parameters were lineariy related to the ultimate strength of the samples. The results of the back propagation network trained with the Weibull parameters is shown in Table 1. The Weibull parameter "b" was found to be the primary hand when the Weibull parameter " $\theta$ " was used in the product " $b * \theta$ " only a slightly better prediction $(-6.28 \%$ error) was made with a relatively large $(-8.39 \%)$ error in the training set. The previous experimental work [1] yielded a worst case error of $5.39 \%$ for the sampled tested. Thus, the neural network approach of mapping the Weibull parameters to the known ultimate strengths was not as accurate as the multivariate statistical analysis.

\begin{tabular}{|c|c|c|c|c|c|c|c|}
\hline \multirow[b]{2}{*}{$\begin{array}{l}\text { Specimen } \\
\text { Number }\end{array}$} & \multirow[b]{2}{*}{$\begin{array}{l}\text { Actual } \\
\text { Strength } \\
\text { (ksi) }\end{array}$} & \multicolumn{2}{|c|}{ b } & \multicolumn{2}{|c|}{$b^{2}=\theta$} & \multicolumn{2}{|c|}{ Event Frequency } \\
\hline & & $\begin{array}{c}\text { Predicted } \\
\text { Strength } \\
\text { (ksi) }\end{array}$ & \% Eтror & $\begin{array}{l}\text { Predicted } \\
\text { Strength } \\
\text { (ksi) } \\
\end{array}$ & \% Error & $\begin{array}{l}\text { Predicled } \\
\text { Surength } \\
\text { (ksi) } \\
\end{array}$ & \% ertor \\
\hline $1+$ & 234.8 & 234.95 & 0.06 & 236.11 & 0.56 & 237.26 & 1.05 \\
\hline $2+$ & 227.6 & 226.50 & -0.48 & 225.09 & -1.10 & 226.88 & -0.32 \\
\hline $3+$ & 237.2 & 236.31 & -0.38 & 235.52 & -0.71 & 233.61 & -1.51 \\
\hline $4+$ & 218.8 & 209.18 & -4.40 & 200.45 & -8.39 & 218.73 & -0.03 \\
\hline $5+$ & 144.0 & 138.49 & -3.55 & 142.72 & -0.89 & 141.81 & -1.52 \\
\hline $6+$ & 176.7 & 182.49 & 3.27 & 180.09 & 1.92 & 178.37 & 0.95 \\
\hline 7 & 224.4 & 204.22 & -8.99 & 224.68 & 0.13 & 228.87 & 1.99 \\
\hline 8 & 215.2 & 213.57 & -0.76 & 220.96 & 5.46 & 216.75 & 0.81 \\
\hline 9 & 233.0 & 232.99 & 0.00 & 236.25 & 1.40 & 241.71 & 3.74 \\
\hline 10 & 192.4 & 201.87 & 4.92 & 180.33 & -6.28 & 186.81 & -2.91 \\
\hline 11 & 138.0 & 143.08 & 3.68 & 138.95 & 0.69 & 140.14 & 1.55 \\
\hline
\end{tabular}

Table 3. Neural network analysis results. 
It was thought that a better ultimate strength prediction could be obtained by training the network with the more detailed event frequency (amplitude) distribution. The individual event count found at each ( $1 \mathrm{~dB}$ ) amplitude interval would serve as the input to the neural network. For this approach a three layer network was employed. The hidden layer allowed the nerwork to characterize the subte variations in the distribution and relate them to the known ultimate strengths in the training set. The network architecture was built around a 23 neuron input layer, an 11 neuron hidden layer, a fully connected bias, and a single output layer neuron for predicting ulimate strengths. The subtle variations found within the amplitude distribution were correlated with the ultimate strength of the training samples (by the hidden layer's weighted connections) to yield a worst case enror of $3.74 \%$.

\subsection{CONCLUSIONS}

The experimental work in this paper demonstrated that a back propagation neural network can be used to predict ultimate strengths in graphite/epoxy tensile specimens by using the event frequency (amplitude) distribution data as the input vectors with their known ultimate strengths as the output vectors. Only the low amplitude portion of the AE data taken up to $25 \%$ of the expected failure strength (from a series of six training specimens) were used in the input training vectors. The hidden layer of the neural network was able to extract and map the subtle fearures of the amplitude distribution data to the known failure strengths of the samples tested. The technique permitted a worst case ultimate strength prediction error of $3.74 \%$. This is somewhat lower than the $5.39 \%$ worst case error from the previous statistical analysis [1].

The neural network was not able to correlate the Weibull distribution parameters of the amplitude data with the ultimate strengths of the samples as well as the multivariate statistical analysis. Due to the smoothing effect of the Weibull model on the event frequency data, the details required to generate accurate ultimate strength predictions were not present. The Weibull distribution modeling parameters are very sensitive to aberrations in the event frequency data set. This leads to the formation of "noisy" input data. Also, with the limited amount of information present in the input vector (" $b$ " and/or " $b * \theta^{\prime \prime}$ ) the neural network has a tendency to memorizing the training data, this then results in higher prediction errors (especially with "noisy" input data).

\subsection{REFERENCES}

1. Walker, J. L. and Hill, E. v. K., "Amplitude Distribution Modeling and Ultimate Strength Prediction of ASTM D-3039 Graphite/Epoxy Tensile Specimens," Proceedings from the Fourth International Svmposium on Acoustic Emission from Composite Materials (AECM-4), The American Society for Nondesuructive Testing, Inc., Columbus OH, July 28-31, 1992.

2. Hill, E. v. K., Isreal, P. L., and Knotts, G. L., "Neural Network Prediction of Aluminum-Lithium Weld Strength from Acoustic Emission Amplitude Data," Materials Evaluaiion, Vol. 51, No. 9, September 1993, pp. 1040-1045, 1051. 